\title{
Experimental study of spin-wave dispersion in Py/Pt film structures in the presence of an interface Dzyaloshinskii-Moriya interaction
}

\author{
A. A. Stashkevich, ${ }^{1,2, *}$ M. Belmeguenai, ${ }^{1}$ Y. Roussigné, ${ }^{1}$ S. M. Cherif, ${ }^{1}$ M. Kostylev, ${ }^{3}$ M. Gabor, ${ }^{4}$ \\ D. Lacour, ${ }^{5}$ C. Tiusan, ${ }^{4,5}$ and M. Hehn ${ }^{5}$ \\ ${ }^{1}$ LSPM (CNRS-UPR 3407), Université Paris 13, Sorbonne Paris Cité, 93430 Villetaneuse, France \\ ${ }^{2}$ ITMO University, International Laboratory "Fundamental Phenomena in Multiferroic Micro and Nanostructures \\ and their Applications in Microelectronics and Microwave Photonics", Saint-Petersburg, Russia \\ ${ }^{3}$ School of Physics, M013, University of Western Australia, Crawley 6009, Western Australia, Australia \\ ${ }^{4}$ Technical University of Cluj-Napoca, Str. Memorandumului No. 28, RO-400114 Cluj-Napoca, Romania \\ ${ }^{5}$ Institut Jean Lamour, CNRS, Université de Lorraine, 54506 Vandoeuvre, France
}

(Received 28 December 2014; revised manuscript received 12 May 2015; published 8 June 2015)

\begin{abstract}
Brillouin light scattering (BLS), complemented by ferromagnetic resonance (FMR) characterization, has been used for studying spin-wave (SW) propagation in $\operatorname{Py}(L) / \mathrm{Pt}(6-\mathrm{nm})$ bilayers of various Py thicknesses $(4 \mathrm{~nm} \leqslant L \leqslant 10 \mathrm{~nm})$. The FMR measurements allowed determination of the pertinent magnetic parameters and revealed the existence of a normal surface anisotropy. A pronounced asymmetry of Damon-Eshbach (DE) wave frequencies has been evidenced by BLS. Therefore, the difference between Stokes and anti-Stokes DE frequencies has been measured versus SW wave number for all the samples. A detailed discussion about the origin of this frequency difference is reported, which concludes that this is due to interface Dzyaloshinskii-Moriya interaction (IDMI).
\end{abstract}

DOI: 10.1103/PhysRevB.91.214409

PACS number(s): 75.30.Ds, 75.30.Gw, 75.70.Cn

\section{INTRODUCTION}

The exchange interaction directly linking adjacent magnetic atoms on the microscopic quantum level is responsible for the most spectacular manifestation of the ferromagnetic ordering: the domain structure. For several decades, it was universally accepted that the exchange interactions are satisfactorily described by the isotropic Heisenberg's model proposed in the late 1920s. However, 30 years later this fundamental item had been revisited and it was shown that in low-symmetry systems an antisymmetric term accounting for the so-called Dzyaloshinskii-Moriya interaction (DMI) has to be added $[1,2]$. This symmetry reduction can occur on the microscopic level in inversion asymmetric crystal fields and considerably influences the behavior of the naturally formed magnetic structures, such as domain walls. Thus DMIs are of major importance in the conversion of the inner structure of the domain wall from a Bloch type to a Néel type with a preferred chirality [3] and can induce chiral structures such as spin spirals and skyrmions [4-11]. In other words, DMI can be regarded as an additional degree of freedom in ferromagnetic behavior, allowing for creating tailor-made chiral magnetic nanostructures otherwise unobtainable.

Nowadays, practically all the applications of thin magnetic films, however different, are unthinkable without nanopatterning, unavoidably leading to breaking of symmetry [12]. This is another means of diversifying potential configurations leading to physical effects where the role of DMIs is instrumental. One such promising possibility is realized via the interfacial Dzyaloshinskii-Moriya interaction (IDMI). It has been a subject of significant interest recently [13-18]. In Ref. [19] a theory, based on a microscopic approach, of spin waves in ferromagnetic atomic monolayers with IDMI was constructed. It has been found that this interaction may lead to significant non-

*Corresponding author: stachkevitch@univ-paris13.fr reciprocity of the spin waves (SWs) in these materials. This SW nonreciprocity yields a difference between the frequencies for the Stokes and anti-Stokes peaks in a Brillouin light-scattering experiment [20] which is proportional to the SW wave number $k_{s w}$. Importantly, the macroscopic formalism employed in a later paper [21] has confirmed earlier theoretical predictions of non-negligible SW reciprocity for films of finite macroscopic thickness $L$ (from ten to several tens of unit cells $a$ for the material). These films are of great technological importance. Also, they make the experimental observation of the IDMIinduced spin-wave nonreciprocity more easily implementable. In terms of general wave physics this can be interpreted as linear spatial dispersion. In optics, it is responsible for optical activity in chiral crystals [22]. Not surprisingly, the symmetric conventional exchange interaction produces quadratic dispersion scaled as $\left(k_{s w}\right)$ [2], an even function of the SW wave number, while the anisotropy engendered by the antisymmetric Dzyaloshinskii-Moriya exchange is characterized by an odd linear functional dependence of the wave frequency on $k_{s w}$.

In the present work, we investigate the IDMI-induced nonreciprocity of SWs propagating in technologically important non-ultrathin $(L>10 a)$ Permalloy $\left(\mathrm{Py}=\mathrm{Ni}_{80} \mathrm{Fe}_{20}\right)$ films. We use the reciprocal-space Brillouin light scattering (BLS) technique to measure the spin-wave dispersion. The Py films were capped with thin platinum $(\mathrm{Pt})$ layers to induce IDMI. Platinum is universally known as a material with a strong spin-orbit interaction, which leads to IDMI. Fert and Levy [23] have shown as early as 1980 that the classical Moriya's asymmetric exchange interactions induced through the purely magnetic two-site mechanism, involving $3 d$ electrons, can be considerably enhanced at the ferromagnetic-heavy metal interface. In the latter case a three-site mechanism involving two magnetic and one nonmagnetic site on which strong spin-orbit interaction occurs is realized [24]. It should be noted that the choice of heavy metals such as platinum is dictated by the fact that the spin-orbit interaction, instrumental in this case, is especially pronounced in them. That is why they are 
widely used in research dedicated to the phenomena where the spin-orbit interaction plays the fundamental role, such as the spin Hall effect [25].

The interface of a ferromagnetic metal with Pt can introduce normal uniaxial interface anisotropy (NUIA) in addition to IDMI. That is why special attention in this paper is given to the separation of contributions of these two effects to the spin-wave frequency. We used the broadband ferromagnetic resonance (FMR) method to this end. Our choice of this method is motivated by the fact that the IDMI contribution scales as $k_{s w}$ [21] and consequently it vanishes in the FMR measurements corresponding to the case $k_{s w}=0$. At the same time, NUIA does not depend explicitly on $k_{s w}$; as a result, its contribution to the FMR frequency is strong enough and can be easily measured with high accuracy [26-28].

The spin-wave frequency dispersion was measured with BLS in a broad range of spin-wave frequencies. The measurements confirmed the recently predicted frequency shift of spin waves due to IDMI [21]. It was also found that the joint action of NUIA and IDMI results in a dispersion curve noticeably curved upward, whereas the Damon-Eschbach dispersion law for isotropic films predicts a negative dispersion curvature for the same film geometry. Furthermore, our theoretical calculations, with the material parameters extracted from the experimental data, demonstrated that for $\mathrm{Pt} / \mathrm{Py}$ bilayer films with Py layer thicknesses below $8 \mathrm{~nm}$ the NUIA contribution to the total nonreciprocal frequency shift is small or negligible with respect to the IDMI contribution.

\section{THEORETICAL CONSIDERATIONS}

As stated in the introduction, for correct interpretation of experimental data for $\mathrm{Pt} / \mathrm{Py}$ films, capability of separation of IDMI and NUIA is important and the experiment has to be set up accordingly. Theoretical consideration as well as experimental verification are carried out in the Damon-Eshbach (DE) [33] configuration, in which case the film is magnetized to saturation by a tangential magnetic field and the investigated spin wave propagates normally to the direction of this field.

Approximate analytical formulas exist for the dipoleexchange dispersion law for DE waves in the presence of normal uniaxial anisotropy [29] and IDMI [21]. The derivation is straightforward, by means of averaging all available effective fields over the film thickness (see e.g., Ref. [21]). Including both effective surface fields into the derivation, one obtains a relation as follows:

$$
\omega\left(k_{s w}\right)=\sqrt{\left[\omega_{H}+\omega_{M}\left(\alpha k_{s w}^{2}+1-N_{u}-P\right)\right]\left[\omega_{H}+\omega_{M}\left(\alpha k_{s w}^{2}+P\right)\right]}+\gamma \mu_{0} D^{*} k_{s w} b / L,
$$

where $D^{*}=2 D /\left(\mu_{0} M\right)$ [19], the effective demagnetizing factor of anisotropy $N_{u}=2 K_{u} / M^{2}$, and $K_{u}=k_{\text {surf }} b / L$. Other parameters in these formulas are $\omega_{H}=\gamma \mu_{0} H, \omega_{M}=\gamma \mu_{0} M$, where $H$ is the applied field, $M$ is the saturation magnetization for the ferromagnetic layer, $\gamma$ is the gyromagnetic coefficient, $\mu_{0}$ is the permeability of vacuum, $\alpha=2 A /\left(\mu_{0} M^{2}\right)$ is the exchange constant, $D$ is the Dzyaloshinskii constant, $K_{u}$ is the constant of normal uniaxial anisotropy, and $P\left(k_{s w}\right)=$ $1-\left[1-\exp \left(-\left|k_{s w}\right| L\right)\right] /\left(\left|k_{s w}\right| L\right)$, where $L$ is the thickness of the ferromagnetic layer and $b$ is the thickness of the interface atomic layer.

The averaging procedure is valid for small spin-wave wave numbers and film thicknesses $\left(k_{s w} L<1\right.$, see, e.g., Ref. [21]), because it neglects effects related to the surface character of the Damon-Eshbach spin waves. For instance, all types of wave nonreciprocities due to the surface character of the wave are neglected, such as potential frequency nonreciprocity due to NUIA. (The nonreciprocities may be included in the second order of the approximate theory, see, e.g., Ref. [30]. However, this goes beyond the scope of the present article with emphasis on experiment.)

Note that the terms describing the two competing mechanisms enter into the formula differently; while the NUIA term is placed under the square root, the IDMI contribution is a linear addition to the SW frequency, in full compliance with the symmetry considerations. The Landau-Lifshitz equation containing a cross product in the right-hand side is naturally diagonalized for circular polarizations. Importantly, the IDMI is characterized by the same symmetry and is also naturally formulated in terms of circular polarizations; as a result it appears in Eq. (1) as a linear term. By contrast, the conventional NUIA surface pinning applies to Cartesian components of the dynamic magnetization that are "mixed" by the abovementioned cross product in the Landau-Lifshitz equation, hence the apparition of the square root.

One notices that in this approximate theory both IDMI and NUIA contributions to Eq. (1) scale as $1 / L$. As it has been pointed out by Soohoo [31] as early as 1963, for films with thicknesses lower than $10 \mathrm{~nm}$ or so, the surface magnetization pinning due to surface effects does not noticeably deform the mode profiles across the film thickness. Thus, as shown by Stamps and Hillebrands [32], the effect of the NUIA is seen as effective bulk anisotropy $K_{u}$, which scales as $1 / L$. The same applies to the IDMI effective field.

The respective interface anisotropy constant $k_{\text {surf }}$ is positive. Furthermore, this constant and the effective field of NUIA are independent from the spin-wave number $k_{s w}$.

There is, however, a fundamental difference between two mechanisms which allows specifying experimental conditions that exclude confusion of the two contributions. From Eq. (1) one sees that for $D=0$ one has $\omega\left(-k_{s w}\right)=\omega\left(k_{s w}\right)$, i.e., no frequency nonreciprocity of spin waves is present for $D=0$. This is not surprising, since the IDMI is excluded and the NUIA constant does not depend directly on $k_{s w}$. Nevertheless, asymmetric NUIA is capable of producing effective nonreciprocity in the propagation of DE modes, but only if the film is thick enough. Naturally, maximum nonreprocity in SW propagation corresponds to maximum asymmetry in localization of surface anisotropy, i.e., to oneside pinning. In thick films the characteristic exponential DE 
spatial asymmetry of the dynamic magnetization distribution across the film [33] for a given SW wave number $k_{s w}$ becomes sufficiently pronounced. Thus it is confined either to the surface with pinning, when traveling in one direction, or to the "free surface" when traveling in the other. As a result, SW propagation becomes nonreciprocal. This effect tends to zero in thin films with homogeneous distribution of dynamic magnetization in a SW mode, which makes the conditions of propagation in two opposite directions identical.

On the contrary, the frequency nonreciprocity due to IDMI exists for any film thickness [as seen from the last term of Eq. (1)], independently from whether IDMI acts as a quasibulk effect [Eq. (1)] or leads to a surface torque and $k_{s w}$-dependent surface (interface) dynamic magnetization pinning [21]. This is due to a direct dependence of the last term on $k_{s w}$, including its sign. The latter means, in particular, that a SW mode traveling in one direction experiences asymmetric one-sided positive pinning, while the mode traveling in the opposite direction is affected by a similar but negative pinning. As a result, the asymmetry of two waves propagating in opposite direction is never identical; however uniform the initial DE profile. The latter consideration favors the IDMI nonreciprocity in thin film. This crucially important point will be revisited later in this paper (see Sec. V, Discussion).

Thus the fundamental difference between IDMI and NUIA resulting from the analysis above can be summarized as follows: whereas the frequency nonreciprocity induced by NUIA grows with $L$, the IDMI-induced frequency nonreciprocity scales approximately as $1 / L$, as clearly seen from Eq. (1). Accordingly, there should exist a range of $L$ values for which the film is already non-ultrathin and whose magnetization dynamics may be studied with standard tools (like MOKE or BLS), but still thin enough to make the NUIA contribution to spin-wave nonreciprocity negligible.

To support this idea, we have carried out numerical simulation of the frequency nonreciprocity due to NUIA $\Delta F=\left[\omega\left(k_{s w}\right)-\omega\left(-k_{s w}\right)\right] /(2 \pi)$ as a function of the SW wave number $k_{s w}$ for films of nanometer-range thicknessesfrom 2 to $12 \mathrm{~nm}$-calculated using the software from Ref. [34]. The latter, unlike Eq. (1) is based on the exact solution of the SW eigenproblem and thus takes into account rigorously all the above-mentioned mechanisms. That is why it will be referred to throughout this paper as an "exact solution." The value of $k_{s w}$ was varied from 3 to $22 \mu \mathrm{m}^{-1}$, which corresponds to the range of spatial frequencies actually covered by the BLS technique. These results are presented in Fig. 1. The effective magnetic parameters (saturation magnetization $M=9.75 \mathrm{kG}$ and $k_{\text {surf }}=0.4 \mathrm{~mJ} / \mathrm{m}^{2}$ ) were extracted from the results of the FMR microwave characterization, described in detail in the first part of Sec. IV, Experimental Results. Since FMR measurement gives no information on the exchange constant, we have used its conventional value $A=10^{-6} \mathrm{erg} / \mathrm{cm}[20,34]$. Importantly, we have assumed that it is a one-side asymmetric pinning, in which case the NUIA-induced SW nonreciprocity is most pronounced, which occurs. It should be mentioned that if spin pinning, however strong, is symmetric (two-side pinning), no frequency nonreciprocity can arise. The applied magnetic field was set equal to that used in the experiment, i.e., to $1 \mathrm{kOe}$. One sees that for thinner films the effect is practically negligible. Thus, in the case of a 4-nm-thick film,

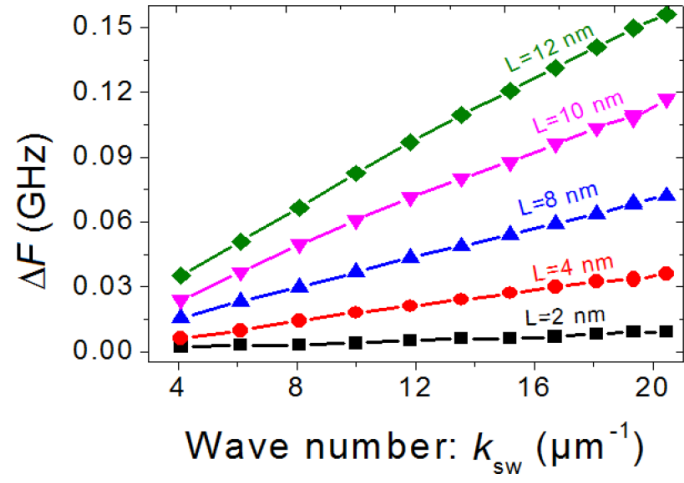

FIG. 1. (Color online) Difference between DE Stokes and antiStokes frequencies of $\operatorname{Py}(L) / \mathrm{Pt}(6-\mathrm{nm})$ bilayers, engendered by asymmetric one-side conventional pinning, as a function of spin-wave number $\left(k_{s w}\right)$ for various $L$. For the numerical calculations, the magnetic parameters extracted from the FMR measurements and indicated in the text were employed. The normal uniaxial easy-axis surface anisotropy constant on the pinned interface was slightly higher than in the experiment: $0.4 \mathrm{~mJ} / \mathrm{m}^{2}$. Hence, the results of the simulation slightly overestimate the NUIA contribution expected for our samples. Symbols refer to the calculated values using the model described in Ref. [34] and solid lines are guides for the eye.

it does not exceed $30 \mathrm{MHz}$. At the same time, the theoretical estimation made in Ref. [21] for a similar 3.55-nm-thick Py film predicts a 500-MHz IDMI-induced SW nonreciprocity (see Fig. 2 in Ref. [21]). Taking into account a slight difference in thickness between the two above-mentioned structures, one would expect something on the order of $400 \mathrm{MHz}$ for the 4-nm film used in our experiments. This ensures at least an order of magnitude of difference between the two contributions. Hence any ambiguity in the interpretation of our experiment results is excluded, given the Dzyaloshinskii-Moriya constant in our case does not differ considerably from that referred to in Ref. [21].

To conclude our preliminary theoretical analysis, the 4-nm Py film (with a 6-nm Pt capping layer) can be considered as the best candidate for investigating the influence of the IDMI on SW propagation in thin films. In this case, the targeted phenomenon-the IDMI-induced nonreciprocity of the SW dispersion - is on the order of $400 \mathrm{MHz}$. This makes it reliably detectable with Brillouin light-scattering spectroscopy. At the same time, the NUIA-related nonreciprocity, regarded here as an unwanted side effect, is weaker by an order of magnitude, which makes the latter negligibly small. In other words, its presence does not compromise the precision of the measuring procedure. It should also be noted that extra caution is to be taken while numerically estimating contributions of the abovementioned mechanisms. On the one hand, the explicit Eq. (1), referred to as the "approximate approach" in the following, accurately describes the IDMI contribution for small wave numbers $k L<1$ or so. Moreover, simple and "user friendly," it provides for a profound physical insight. At the same time, it is entirely inadequate for numerical simulations of the NUIAinduced nonreciprocal effects, especially in thick films, and is to be replaced by the above-mentioned "exact solution" [34].

The scaling of $\Delta F$ induced as $k_{s w}$ dictates the choice of measurement techniques: Brillouin light scattering, giving 
access to SW wave numbers as large as $20 \mu \mathrm{m}^{-1}$, can be used as a principal experimental tool in this study - to probe spinwave dispersion. Furthermore, because the IDMI contribution to the frequency of the ferromagnetic resonance (corresponds to a spin wave with $k_{s w}=0$ ) vanishes [21] but the NIUA contribution remains strong [see Eq. (1)], FMR can be used as a supplementary technique of sample characterization, allowing direct experimental estimation of the strength of NUIA. These data are, as has been shown earlier, of great importance for reliable separation of the two contributions (IDMI and NUIA) to spin-wave nonreciprocity.

In other words, in this work we employ BLS as the main instrument to study $\mathrm{SW}$ propagation in $\mathrm{Pt} / \mathrm{Py}$ films with $\mathrm{Py}$ thicknesses 4-10 nm, supported by FMR microwave sample characterization.

\section{SAMPLES AND EXPERIMENTAL SETUPS}

Permalloy $\left(\mathrm{Py}=\mathrm{Ni}_{80} \mathrm{Fe}_{20}\right)$ thin films with thickness varying from 4 to $10 \mathrm{~nm}(L=4,6$, and $10 \mathrm{~nm})$ were grown by UHV sputtering in Ar pressure of 5 mTorr on Si substrates and then capped with a 6-nm-thick Pt layer. The role of the 6-nm Pt top layer was to boost the IDMI on the Py/Pt interface; platinum is universally known as a material with a strong spin-orbit interaction.

As pointed out earlier, our experimental procedure was based on a two-level approach. The BLS experiments give access to spin-wave modes with nonzero wave-vector values ranging from 0.2 to $20 \mu \mathrm{m}^{-1}$, which is essential in investigations of $k_{s w}$-dependent IDMI-induced pinning. At the same time it excludes the important point of $k_{s w}=0$. That is why we used a microstrip ferromagnetic resonance (MS-FMR) technique [28] for preliminary microwave characterization. Importantly, for purely technical reasons MS-FMR measurements as a function of the direction of the applied magnetic field are easier to implement. In this sense it is more flexible than BLS and was used to determine the pertinent magnetic parameters of the samples [28].

In our MS-FMR measurements the sample is mounted on a $0.5-\mathrm{mm}$-wide microstrip line. One port of the line was connected to the microwave generator; its second port was connected to a microwave (Schottky) diode whose output was fed into a lock-in amplifier. A magnetic field was applied either in the film plane along different directions with respect to the sample edges or perpendicular to the sample plane. A small-amplitude modulating ac magnetic field was applied along the direction of the strong field. The lock-in amplifier was referenced by the modulating signal.

In the BLS spectroscopy the spin waves traveling in the film inelastically scatter the light provided by an incident monochromatic beam. The frequency shift is analyzed using a $2 \times 3$ pass Fabry-Perot interferometer, which typically gives access to the $3-300 \mathrm{GHz}$ spectral frequency range. For the backscattering configuration used, the investigated wave vector lies in the plane of incidence and its amplitude is equal to $k_{s w}=4 \pi \sin (\theta) / \lambda$ ( $\theta$ is the angle of incidence and $\lambda$ is the wavelength of the illuminating laser, $\lambda=532 \mathrm{~nm}$ ), swept in interval from 0.2 to $20 \mu \mathrm{m}^{-1}$ through the rotation of the sample around a planar axis. The magnetic field was applied perpendicular to the incidence plane, thus probing SW propagating along the in-plane direction perpendicular to the applied field (DE configuration).

High-frequency resolution is of extreme importance in this study, which is only possible with very high-quality spectra. To attain it the following experimental procedure has been adopted. First, we have chosen Permalloy as the material for the ferromagnetic layer. It is universally known as having low losses at microwaves, which provides for narrow well-defined BLS spectral lines. Typically, spectra of acceptable quality are obtainable within $1 \mathrm{~h}$ of signal collection. However, in our case it is not sufficient. It is common knowledge that the BLS intensity diminishes for high angles of incidence and high values of the saturating magnetic field. That is why to take the BLS measurements, the magnetic field value $H$ was as low as $\pm 1 \mathrm{kOe}$. Moreover, the accumulation time was adapted accordingly; it varied from several hours for small angles up to $12 \mathrm{~h}$ for the incidence close to grazing. Importantly, with spectral lines thus recorded Lorenzian fitting lead to coincidence with the experiment close to graphic resolution.

Using low working fields has another advantage; it also means relatively low frequencies of the investigated $\mathrm{SW}$ modes (around $10 \mathrm{GHz}$ ), which justifies narrowing the range of frequency sweeping in the BLS experiment. The latter is important, since in this way one minimizes the frequency step between two adjacent channels (133 MHz).

It should also be emphasized that essential in this study is not measuring the absolute value SW mode frequencies, but the difference between two closely spaced values (Stokes and anti-Stokes). Hence a number of otherwise-inevitable instrumental errors are excluded, e.g., uneven rate of frequency sweeping and zero point drifting. All this yields well-defined spectra where the difference in the BLS line position can be determined with accuracy on the order of $\pm 75 \mathrm{MHz}$. Such accuracy is by no means unusual for Brillouin spectroscopy. For instance, in a recent paper from NIST [35], dedicated to BLS investigation of IDMI in similar Py/Pt structures, the authors claim having revealed a nonreciprocity of only $0.25 \mathrm{GHz}$, i.e., approximately 2 times smaller than in our case. Interestingly, their samples were much thinner $[\mathrm{Py}(2 \mathrm{~nm})$ and $\operatorname{Py}(1.3 \mathrm{~nm})]$, which must lead, at least theoretically, to a proportional increase in the effective DMI constant. In other words, the expected values should amount to roughly 0.8 and 1.2 GHz, correspondingly, which is not the case.

In our work the extracted difference between the Stokes and anti-Stokes frequencies $(\Delta F)$ has been studied as a function of $k_{s w}$. Moreover, in order to be on the safe side, we have reverified these results by taking advantage of the symmetry of the investigated effect $\Delta F(-H) \approx-\Delta F(H)$; it had been rigorously shown in Ref. [21] that the difference between $|\Delta F(-H)|$ and $|\Delta F(H)|$ is negligible with respect to $|\Delta F(H)|$ for thicknesses below $10 \mathrm{~nm}$. This relation allows double-checking the measured value of nonreciprocity. To this end for each value of $k_{s w}$ we took measurements for $\mathrm{H}=$ +1000 Oe and -1000 Oe and compared the obtained results.

\section{EXPERIMENTAL RESULTS}

As seen from (1), for $k_{s w}=0$ (corresponds to $P=0$ ), the effect of NUIA reduces to renormalization of the saturation 


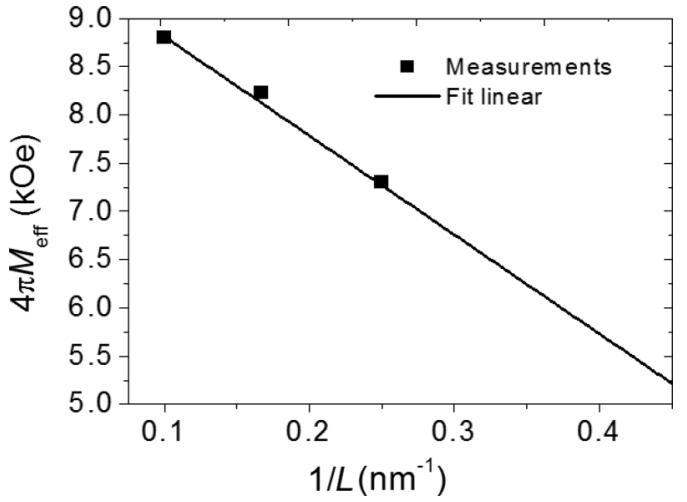

FIG. 2. Effective magnetization as a function of the Py inverse thickness $1 / L$ of the $\operatorname{Py}(L) / \operatorname{Pt}(6-n m)$ bilayers. Symbols refer to the MS-FMR measurements and solid line is the linear fit.

magnetization. Thus an effective saturation magnetization,

$$
M_{\mathrm{eff}}=M-\frac{H_{u}}{4 \pi}=M-\frac{k_{\text {surf }}}{2 \pi M L},
$$

can be introduced, where $H_{\boldsymbol{u}}$ is the effective field of NUIA (averaged over the film thickness, as explained above).

In Fig. 2 are presented the effective magnetization ( $4 \pi M_{e f f}$ ) values extracted using Eq. (2) from the best fit of the in-plane field dependencies of the FMR resonance frequencies as a function of $1 / L$. This approach has been suggested in Ref. [32] and is largely used in the literature [26-28]. The slope of this dependence gives the $k_{\text {surf }}$ value, while the horizontal axis intercept gives the saturation magnetization close to its conventional bulk value $4 \pi M=0.975 \mathrm{~T}$. The obtained NUIA constant is equal to $k_{\text {surf }}=0.4 \mathrm{~mJ} / \mathrm{m}^{2}$. Unfortunately, for films with $L \leqslant 10 \mathrm{~nm}$ it is impossible to specify the contribution of each particular surface to this overall figure, but the sign of the slope of this dependence suggests that this anisotropy is of the easy-axis type, which is typical for an interface with Pt.

It is worth mentioning that besides the determination of $k_{\text {surf }}$, FMR with a perpendicular applied magnetic field allowed us to accurately determine the gyromagnetic factor estimated to be $\gamma=1.854 \times 10^{11} \mathrm{~T}^{-1} \mathrm{~s}^{-1}$, which is slightly greater than some of the earlier published figures. (See, for example, Ref. [36], dedicated to the study of the mobility of domain wall motion, where the reported value is $1.79 \times 10^{11} \mathrm{~T}^{-1} \mathrm{~s}^{-1}$.) At the same time, in a recent paper [37] addressing precise determination of the spectroscopic $g$ factor in Permalloy by broadband ferromagnetic resonance measurements, the following value has been reported: $2.109 \pm 0.003$. The latter corresponds to $\gamma=1.855 \times 10^{11} \mathrm{~T}^{-1} \mathrm{~s}^{-1}$, which is in excellent agreement with our measurements. Furthermore, our FMR characterization revealed that a small in-plane uniaxial anisotropy is present in the samples (maximal anisotropy field of $8 \mathrm{Oe}$ ).

The FMR data also deliver information on the microwave quality of the films. Table I shows the FMR peak-to-peak linewidths $\Delta H^{P P}$, at resonance fields of $1000 \mathrm{Oe}$, for all the samples. From this table, one sees that the resonance linewidth drops with an increase in $L$. This behavior is consistent with pronounced contribution of the spin-pumping effect to the resonance linewidth $[38,39]$. As follows from the former paper,
TABLE I. FMR and BLS linewidths of $\mathrm{Py}(L) / \mathrm{Pt}(6-\mathrm{nm})$ bilayers measured at applied field of $1 \mathrm{kOe}$.

\begin{tabular}{lccc}
\hline \hline Film thickness $L(\mathrm{~nm})$ & 4 & 6 & 10 \\
\hline $\begin{array}{l}\text { Peak-to-peak FMR } \\
\text { linewidth } \Delta H^{P P} \text { at } 1000 \text { Oe }(\mathrm{Oe})\end{array}$ & 75 & 55 & 45 \\
$\begin{array}{l}\text { BLS FWHM linewidth at } \\
\text { 1000 Oe }(\mathrm{GHz})\end{array}$ & 0.87 & 0.77 & 0.69 \\
\hline
\end{tabular}

the spin-pumping contribution to the resonance linewidth should be the largest for $L=4 \mathrm{~nm}$ and should gradually reduce with an increase in $L$. For $L=10 \mathrm{~nm}$, one may expect that the spin-pumping contribution is small with respect to the intrinsic magnetic losses of the Py layer.

The last row of Table I shows the measured widths of the BLS peaks (FWHM). One sees that, similar to the FMR linewidth, the BLS linewidth drops with an increase in $L$. This may suggest that spin pumping also affects the BLS linewidth. It should be mentioned that other mechanisms, such as surface defect-associated relaxation, in thinner films can also contribute to the observed increase in damping.

As expected [see Eq. (1)], the smallest $\Delta F$ was observed for the thickest sample $-L=10 \mathrm{~nm}$. More specifically, we obtained $\Delta F=0$, even for the largest light incidence angle $\theta=60^{\circ}$ [Fig. 3(b)]. Importantly, the same result was obtained for both $H=+1000$ Oe and $H=-1000$ Oe. This demonstrates its reproducibility. The measurements taken for the $L=6 \mathrm{~nm}$ sample [see Fig. 3(a)] showed that the
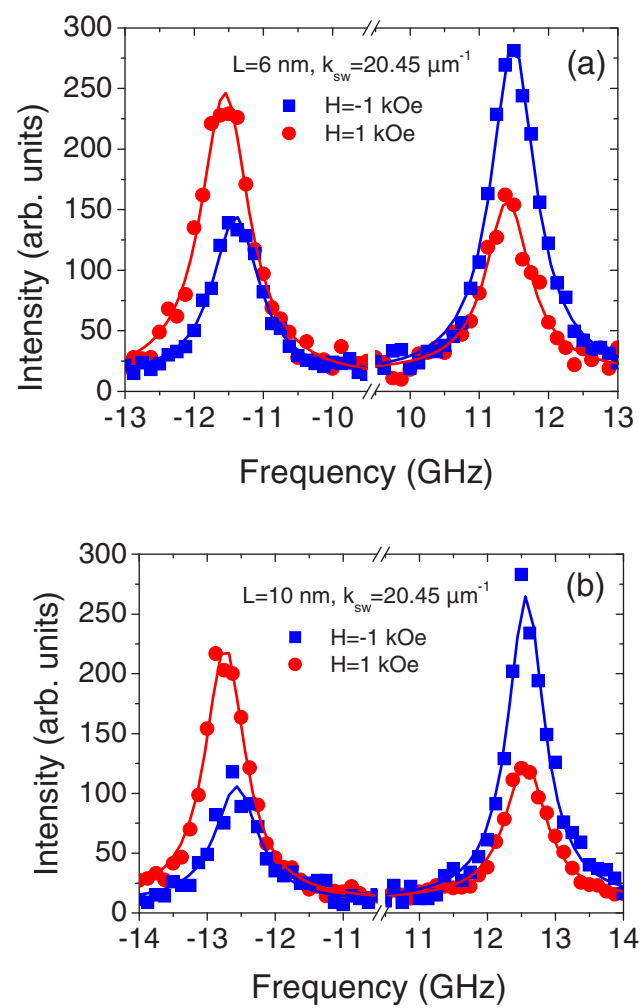

FIG. 3. (Color online) Measured BLS spectra at two different applied field values $(H= \pm 1 \mathrm{kOe})$ for the $\mathrm{Py}(6-\mathrm{nm}) / \mathrm{Pt}(6-\mathrm{nm})$ (a) and $\mathrm{Py}(10-\mathrm{nm}) / \operatorname{Pt}(6-\mathrm{nm})$ (b) films for $k_{s w}=20.45 \mu \mathrm{m}^{-1}$. Symbols refer to the experimental data and solid lines are the Lorentzian fits. 

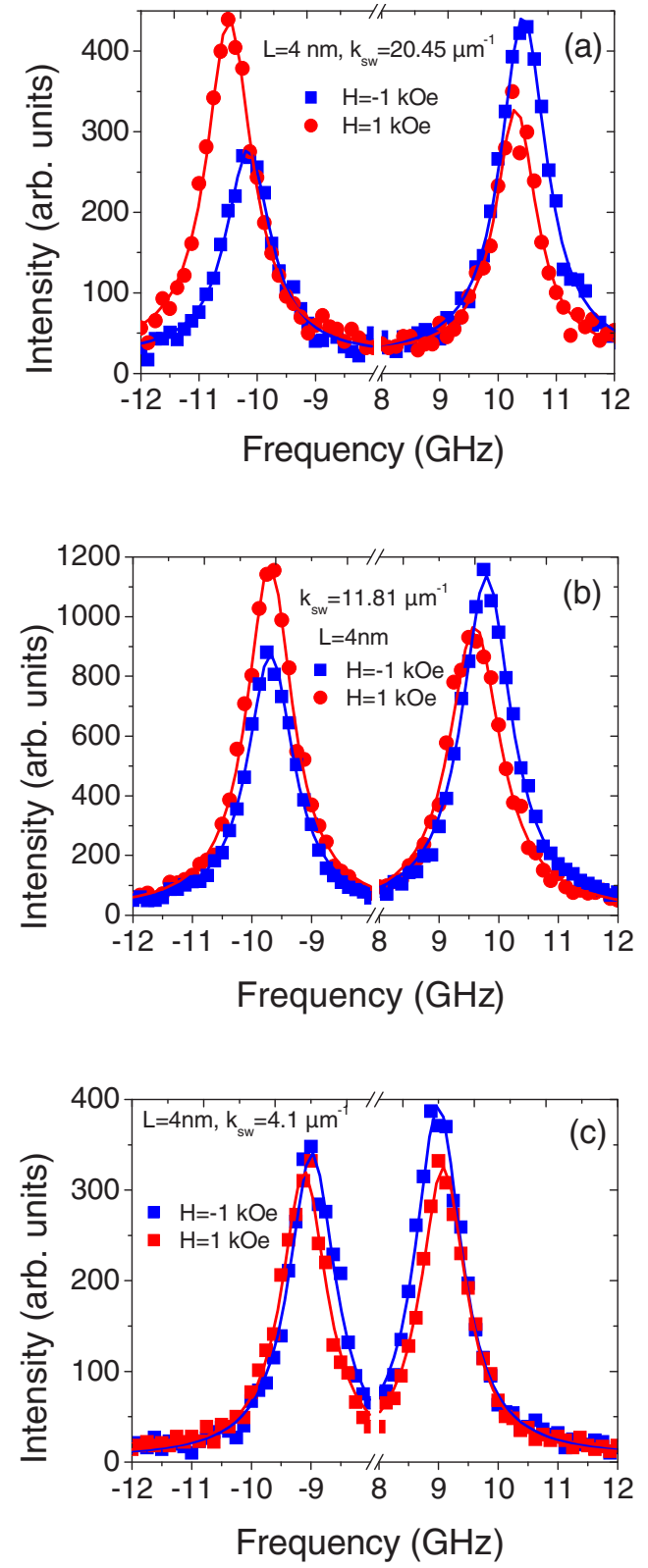

FIG. 4. (Color online) BLS spectra measured for Py(4-nm)/ $\mathrm{Pt}(6-\mathrm{nm})$ bilayer at two different applied field values $(\mathrm{H}= \pm 1 \mathrm{kOe})$ and for three characteristic wave numbers $k_{s w}$ : (a) $k_{s w}=20.45 \mu \mathrm{m}^{-1}$, (b) $k_{s w}=11.81 \mu \mathrm{m}^{-1}$, and (c) $k_{s w}=4.1 \mu \mathrm{m}^{-1}$. Symbols refer to the experimental data and solid lines are the Lorentzian fits.

nonreciprocity $\Delta F$ for the maximum value of $k_{s w}=$ $20.45 \mu \mathrm{m}^{-1}$ (corresponding to $\theta=60^{\circ}$ ) equals approximately $150 \mathrm{MHz}$ and is also the same for $+H$ and $-H$, as presented in Fig. 3(a). For smaller incidence angles, a tendency of a decrease in $\Delta F$ with a decrease in $\theta$ is traceable in the raw BLS data for this sample. However, the value of $\Delta F$ could not be reliably extracted from these traces because of its smallness with respect to the resonance linewidth.

The 4-nm-thick film demonstrated a well-resolved frequency shift $\Delta F$ for a broad range of the angles of light incidence. These data are displayed in Fig. 4. More specifically, in Figs. 4(a)-4(c) are given BLS spectra of the DE mode for $k_{s w}=20.45,11.81$, and $4.1 \mu \mathrm{m}^{-1}$, corresponding to angles of incidence $\theta=60^{\circ}, 30^{\circ}$, and $10^{\circ}$, respectively. The results for $+H$ and $-H$ are very close, as one can see from this figure.

The evolution of the frequency nonreciprocity with $k_{s w}$ can be easily traced; it is clearly visible in Fig. 4(a), becomes less pronounced in Fig. 4(b), and is practically nonexistent in Fig. 4(c). This tendency is illustrated in more detail in Fig. 5, where the dependence of the frequency shift $\Delta F$ (in gigahertz) due to IDMI for $k_{s w}>0$ (black solid line for theory and symbols for experiment) and $k_{s w}<0$ (red solid line for theory) is shown for the $L=4 \mathrm{~nm}$ sample. The dashed lines represent the respective theoretical values for $k_{\text {surf }}=0.33 \mathrm{~mJ} / \mathrm{m}^{2}$ and $D=0$. As mentioned earlier, theory confirms the fact that the IDMI-induced nonreciprocity strongly dominates over the NUIA contribution. Note that the latter value, referred to as "corrected" in the following, differs from the preliminary one obtained by application of Eq. (2) to the data presented in Fig. $2\left(0.4 \mathrm{~mJ} / \mathrm{m}^{2}\right)$. The origin of this correction will be dealt with in the next section.

\section{DISCUSSION}

The processed BLS data from Fig. 5 were fitted with the theory from Ref. [21]. We used the rigorous numerical treatment of the spin-wave dispersion rather than the approximate formula (1), since it was found that for the largest spin-wave number accessible in the experiment, $k_{s w}=20.45 \mu \mathrm{m}^{-1}$, the rigorously calculated theoretical dependence $\Delta F$ vs $1 / L$ is not perfectly linear for the range of Py thicknesses available in our experiment. This demonstrates the importance of the dynamic dipole field leading to surface-wave character for large wave numbers for our thickness range (see Sec. II). In order to make these fits, we included the effective field of NUIA into the program code. This is a straightforward procedure, fully analogous to inclusion of the IDMI effective field. In the following, we refer to this modified code as "the theory from Ref. [21]."

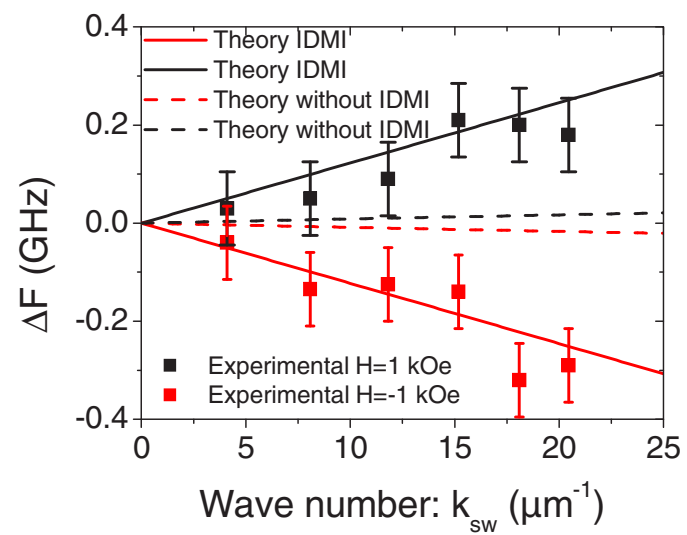

FIG. 5. (Color online) Wave-number $\left(k_{s w}\right)$ dependence of the frequency difference $\Delta F$ (in gigahertz) for $\mathrm{Py}(4-\mathrm{nm}) / \mathrm{Pt}(6-\mathrm{nm})$ bilayer, corresponding to IDMI-induced nonreciprocity: theory versus experiment. The solid lines refer to the theoretical values using the model described in Ref. [21] with IDMI constant $D=1.2 \mathrm{~mJ} / \mathrm{m}^{2}$ and magnetic parameters extracted from the FMR measurements and indicated in the text. The dashed lines represent the corresponding theoretical values due the surface anisotropy with $k_{\text {surf }}=0.33 \mathrm{~mJ} / \mathrm{m}^{2}$. 




FIG. 6. (Color online) Effect of IDMI on SW dispersion for $\mathrm{Py}(4-\mathrm{nm}) / \mathrm{Pt}(6-\mathrm{nm})$ bilayer. Theoretical and experimental mean values are given by a green solid line and filled black squares, respectively. Dashed lines (blue and red) indicate splitting due to IDMI. The simulations were carried out using the model described in Ref. [21] with IDMI constant $D=1.2 \mathrm{~mJ} / \mathrm{m}^{2}, k_{\text {surf }}=0.33 \mathrm{~mJ} / \mathrm{m}^{2}$, and magnetic parameters extracted from the FMR measurements and indicated in the text.

Our idea was to fit the sets of experimental data from Figs. 2 and 5 simultaneously with the same set of parameters from Fig. 2. Since Fig. 1 predicts that the contribution of NUIA to $\Delta F$ for the $L=4 \mathrm{~nm}$ film should be almost negligible, the first step was to fit the data from Fig. 5. The best agreement between the theory and the experiment was attained with the IDMI constant $D=1.2 \mathrm{~mJ} / \mathrm{m}^{2}$, which is of realistic order of magnitude $[3,40]$. The dashed lines are theoretical estimations of a similar nonreciprocity due to NUIA in the configuration when it is most pronounced, namely, the entirely asymmetric case with only one interface pinned. From these traces, one sees that the effect of NUIA is too small to undermine the reliability of our observations and hence most of the experimentally measured $\Delta F$ should be due to IDMI.

To make our study comprehensive, we have also investigated the influence of surface pinning on the overall run of the dispersion curve. In Fig. 6 is plotted an average value $\left[F\left(+k_{s w}\right)+F\left(-k_{s w}\right)\right] / 2$, both experimental and theoretical (solid curve). For reference, with dashed lines is indicated IDMI-induced splitting. Since three major characteristics of the dispersion curve (its slope, its curvature, and the value of the FMR frequency $\left.\left[\omega\left(k_{s w}=0\right)\right]\right)$ are to be fitted simultaneously; this procedure provides valuable independent information on actual values of intrinsic magnetic parameters of the sample under investigation otherwise unobtainable. The values which fit the experimental data the best turned out to be $A=0.7 \times 10^{-6} \mathrm{erg} / \mathrm{cm}$ and $k_{\text {surf }}=0.33 \mathrm{~mJ} / \mathrm{m}^{2}$. Interestingly, the latter figure is noticeably smaller than the effective value obtained through FMR measurements $\left(0.4 \mathrm{~mJ} / \mathrm{m}^{2}\right)$. In other words, the preliminary effective value of the surface anisotropy overestimates this effect and, consequently, the NUIA-induced contribution to the nonreciprocity is also overestimated. This, in its turn, makes even less realistic any ambiguity in the interpretation of the origin of the observed nonreciprocity.

Another interesting feature related to surface pinning is revealed through the distortion of the dispersion curve in Fig. 6.
TABLE II. Experimental and theoretical resonance frequencies of $\operatorname{Py}(L) / \operatorname{Pt}(6-\mathrm{nm})$ bilayers corresponding an applied field of $1 \mathrm{kOe}$.

\begin{tabular}{lccc}
\hline \hline Film thickness $L(\mathrm{~nm})$ & 4 & 6 & 10 \\
\hline Experimental resonance field $(\mathrm{Oe})$ & 983 & 1000 & 974 \\
$\begin{array}{l}\text { Experimental resonance } \\
\quad \text { frequency }(\mathrm{GHz})\end{array}$ & 8.5 & 9.00 & 9.0 \\
$\quad \begin{array}{l}\text { Theoretical resonance } \\
\quad \text { frequency } \omega\left(k_{s w}=0\right) /(2 \pi)(\mathrm{GHz})\end{array}$ & 8.485 & 9.01 & 9.191 \\
\hline \hline
\end{tabular}

The classical DE formula [33], derived in the magnetostatic approximation, is incapable of correctly reproducing the experimentally observed dispersion. While the Damon-Eschbach dispersion curve is characterized by a negative curvature (the line is curved down), the experimental and theoretical clearly demonstrate that the dispersion is curved up. This is an indication that the wave of magnetization propagating in the investigated structure is of a more complex dipole-exchange nature [29]. This has been fully confirmed by our numerical calculations, also presented in Fig. 6.

The last step of the fitting procedure was to check which value $k_{\text {surf }}=0.4 \mathrm{~mJ} / \mathrm{m}^{2}$ or $k_{\text {surf }}=0.33 \mathrm{~mJ} / \mathrm{m}^{2}$ actually fits the last experimental point for $k_{s w}=0$ provided by FMR measurements. Not surprisingly, both rigorous theories [34,21] showed that it is the value of $k_{\text {surf }}=0.33 \mathrm{~mJ} / \mathrm{m}^{2}$ that correctly reproduces the slope of Fig. 2 and the agreement of the theory and the experimental FMR frequencies is good (Table II).

This result suggests that the films with thicknesses around $4 \mathrm{~nm}$ cannot be regarded as thin enough to fully justify the fits of the experimental data with $1 / L$ law [32] based on the approximate Eq. (2). The difference between the accurate and the approximate value of $k_{\text {surf }}$ reaches $20 \%$. Naturally, this discrepancy will grow with the film thickness, which is of undeniable importance for the theoretical interpretation of our experimental results obtained on thicker structures. Since the scope of the present paper is IDMI and BLS studies but not NUIA and FMR, these data will be published elsewhere, as going well beyond the scope of the present paper.

Since verified numerical formalisms and reliable magnetic parameters $\left(D, A\right.$, and $\left.k_{\text {surf }}\right)$ extracted during the preceding steps are available now, we can proceed to calculating $\Delta F$ for the two thicker films. The numerical simulations showed that $\Delta F\left(k_{s w}=20.45 \mu \mathrm{m}^{-1}\right)$ is 120 and $-36 \mathrm{MHz}$ for $L=6$ and $10 \mathrm{~nm}$, respectively. The first one is in fair agreement with the experiment $(150 \mathrm{MHz}$, see Sec. IV); the latter $(-36 \mathrm{MHz})$ is much smaller than the BLS resolution, $\pm 75 \mathrm{MHz}$, and is hence undetectable with the BLS method.

Interestingly, the simulations showed that $\Delta F$ of $-36 \mathrm{MHz}$ for $L=10 \mathrm{~nm}$ is "composed" of two almost equal contributions with opposite signs: $+92 \mathrm{MHz}$ of the shift due to IDMI and $-133 \mathrm{MHz}$ of the shift due to NUIA. (Note that the sum of the latter two values is not exactly $-36 \mathrm{MHz}$.) That is why one should not be surprised to have this sign inversion of the frequency asymmetry; this simply means that in thicker films the NUIA mechanism becomes predominant. Thus the vanishing experimental value of $\Delta F$ for $L=10 \mathrm{~nm}$ can be regarded as an independent confirmation of the positive sign of $D$. Indeed, if $D$ were negative, the total $\Delta F$ would be about $230 \mathrm{MHz}$, which would be easily measurable. 
(a) Surface anisotropy: thick film



(b) Surface anisotropy: thin film

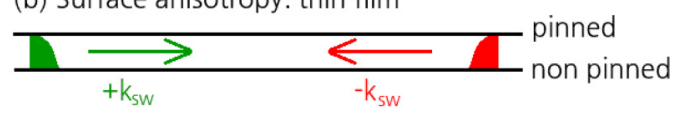

(c) IDMI anisotropy: thin film

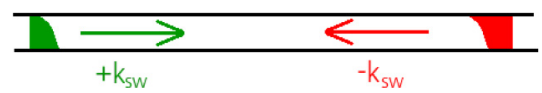

FIG. 7. (Color online) Schematic illustration of the SW profiles induced by two different mechanisms (IDMI and NUIA) in thick and thin films yielding SW nonreciprocity.

Here it is worth mentioning that the experimental dependence of $\Delta F\left(k_{s w}=20.45 \mu \mathrm{m}^{-1}\right)$ vs $1 / L$ is not a straight line (see the data in the end of Sec. IV). The results of the numerical simulation shown above do not obey a linear law either. The latter correlates with the development of the significant frequency shift due to NUIA for $L=10 \mathrm{~nm}$. This shows that the dynamic dipole field of the Damon-Eshbach spin wave is the main contributor to the nonlinearity of $\Delta F$ vs $1 / L$.

To explain this behavior, one has to go beyond the picture of the effective bulk anisotropies of Eq. (1) and consider them as pure surface effects (Fig. 7). The latter is the essence of the rigorous numerical approaches [34,21]. More specifically, in the upper panels (a) and (b) are shown SW profiles for the Py layer in the case of conventional one-sided (only spins at the upper Py/Pt interface pinned) NUIA pinning in (a) a thick and (b) a thin film. The conventional pinning is independent of the $k_{s w}$ sign and thus produces the same effect on the profiles of the two SWs propagating in opposite directions (green for $+k_{s w}$ and red for $-k_{s w}$ ), namely, a characteristic drop of the dynamic magnetization in the vicinity of the pinned upper interface. In thicker films, $2\left|k_{s w}\right| L$ becomes comparable to 1 , which results in classical asymmetric exponential DE profiles which are developed due to considerable contribution of the dipole field to the total dynamic effective field. Importantly, the one corresponding to $+k_{s w}$ is maximal at the upper interface while the other is minimal. One-sided pinning leads to two different profiles and hence non-negligible frequency nonreciprocity. In thin films [Fig. 7(b)], $2\left|k_{s w}\right| L \ll 1$ and, consequently, the DE wave profile is practically uniform [Fig. 7(b)]. As a result, the red and green profiles are identical, which explains absence of any nonreciprocity.

By contrast, one-sided IDMI pinning changes its sign with inversion of the spin-wave wave-vector direction which, when applied to an otherwise perfectly uniform DE profile in a thin film [Fig. 7(c)], renders both profiles (red and green) asymmetric but in a different way. It is a characteristic drop in dynamic magnetization near the pinned surface for $+k_{s w}$ and a no less characteristic rise for $-k_{s w}$ and hence, the nonreciprocity.

\section{CONCLUSION}

While the role of the Dzyaloshikskii-Moriya (DMI) interactions in the conversion of the domain walls with a preferred chirality as well as in creation of chiral structures such as spin spirals and skyrmions has been under intensive investigation during the last decade, their influence on magnetic dynamics remains largely unknown. In this paper we have addressed the most spectacular manifestation of the symmetry of interface DMIs in the behavior of magnetic excitations in structured thin ferromagnetic films. More specifically, we have experimentally observed the nonreciprocity of spinwave propagation induced by interface Dzyaloshinskii-Moriya interaction (IDMI) predicted theoretically earlier. To verify these predictions we used $\mathrm{Py} / \mathrm{Pt}(6-\mathrm{nm})$ bilayers of various Py thicknesses. Since the IDMI effect is explicitly proportional to the $\mathrm{SW}$ wave number $k_{s w}$, the characteristic nonreciprocity $\Delta F$ was studied by Brillouin light scattering (BLS), while ferromagnetic resonance (FMR) was used as a backing technique for microwave characterization. As predicted theoretically, $\Delta F$ turned out to be linear in $k_{s w}$, reaching a value of $300 \mathrm{MHz}$ for $k_{s w}=20.45 \mu \mathrm{m}^{-1}$ in the 4-nm thick-sample. The latter allows estimating the Dzyaloshinskii constant as $D=1.2 \mathrm{~mJ} / \mathrm{m}^{2}$, which confirms the numerical values obtained earlier by means of "static" methods.

The theoretical analysis has revealed the importance of reliable numerical estimation of SW nonreciprocity originating from conventional surface pinning. We have shown that, while in ultrathin films (e.g., $4 \mathrm{~nm}$ ) this contribution is negligibly small, in the intermediate range $(8-10 \mathrm{~nm})$ it becomes comparable to that induced through IDMI. The latter provided us with a valuable instrument of identification of the sign of the Dzyaloshinskii constant. Thus the constructive "in-phase" superposition of the two contributions is reliably detectable with the BLS technique. On the other hand, the destructive "antiphase" superposition corresponds to a vanishing resultant $\Delta F$, which has been actually observed in the experiment.

\section{ACKNOWLEDGMENTS}

Financial support from C'nano - Région Ile-de-France (IMADYN project), FEDER, the Region Lorraine, the Grand Nancy, the Australian Research Council, The University of Western Australia, the Government of the Russian Federation (Grant 074-U01), and the Government of Romania (the Exploratory Research Project SPINTAIL: PN-II-ID-PCE-20124-0315, No. 23/29.08.2013, Project SPINTRONIC: POS CCE ID. 574, code SMIS-CSNR 12467, and TUCN-MADSPIN Research Project) is acknowledged.
[1] I. E. Dzyaloshinskii, Sov. Phys. JETP 5, 1259 (1957).

[2] T. Moriya, Phys. Rev. 120, 91 (1960).
[3] A. Thiaville, S. Rohart, E. Jué, V. Cros, and A. Fert, Europhys. Lett. 100, 57002 (2012). 
[4] U. K. Rößler, A. N. Bogdanov, and C. Pfleiderer, Nature (London) 442, 797 (2006).

[5] M. Uchida, Y. Onose, Y. Matsui, and Y. Tokura, Science 311, 359 (2006).

[6] S. Mülbauer, B. Binz, F. Jonietz, C. Pfleiderer, A. Rosch, A. Neubauer, R. Georgii, and P. Böni, Science 323, 915 (2009).

[7] S. D. Yi, S. Onoda, N. Nagaosa, and J. H. Han, Phys. Rev. B 80, 054416 (2009).

[8] X. Z. Yu, Y. Onose, N. Kanazawa, J. H. park, J. H. Han, Y. Matsui, N. Nagaosa, and Y. Tokura, Nature (London) 465, 901 (2010).

[9] X. Z. Yu, N. Kanazawa, Y. Onose, K. Kimoto, W. Z. Zhang, S. Ishiwata, Y. Matsui, and Y. Tokura, Nat. Mater. 10, 106 (2011).

[10] S. Heinze, K. von Bergmann, M. Menzel, J. Brede, A. Kubetzka, R. Wiesendanger, G. Bihlmayer, and S. Blügel, Nat. Phys. 7, 713 (2011).

[11] S. X. Huang and C. L. Chien, Phys. Rev. Lett. 108, 267201 (2012).

[12] A. R. Fert, Mater. Sci. Forum 59-60, 439 (1990).

[13] G. Chen, J. Zhu, A. Quesada, J. Li, A. T. N'Diaye, Y. Huo, T. P. Ma, Y. Chen, H. Y. Kwon, C. Won, Z. Q. Qiu, A. K. Schmid, and Y. Z. Wu, Phys. Rev. Lett. 110, 177204 (2013).

[14] S. Emori, U. Bauer, S.-M. Ahn, E. Martinez, and G. S. D. Beach, Nat. Mater. 12, 611 (2013).

[15] K.-S. Ryu, L. Thomas, S.-H. Yang, and S. S. P. Parkin, Nat. Nanotechnol. 8, 527 (2013).

[16] L. Udvardi and L. Szunyogh, Phys. Rev. Lett. 102, 207204 (2009).

[17] A. T. Costa, R. B. Muniz, S. Lounis, A. B. Klautau, and D. L. Mills, Phys. Rev. B 82, 014428 (2010).

[18] Kh. Zakeri, Y. Zhang, T.-H. Chuang, and J. Kirschner, Phys. Rev. Lett. 108, 197205 (2012).

[19] J.-H. Moon, S.-M. Seo, K.-J. Lee, K.-W. Kim, J. Ryu, H.-W. Lee, R. D. McMichael, and M. D. Stiles, Phys. Rev. B 88, 184404 (2013).

[20] B. Hillebrands, in Modern Techniques for Characterization of Magnetic Materials, edited by Yimei Zhu (Springer-Verlag, Berlin, 2005).

[21] M. Kostylev, J. Appl. Phys. 115, 233902 (2014).
[22] V. M. Agranovich and V. L. Ginzburg, Spatial Dispersion in Crystal Optics and the Theory of Excitons (InterScience Publishers, New York, 1966).

[23] A. Fert and P. M. Levy, Phys. Rev. Lett. 44, 1538 (1980).

[24] A. Crépieux and C. J. Lacroix, Magn. Magn. Mater. 182, 341 (1998).

[25] V. E. Demidov, S. Urazhdin, E. R. J. Edwards, and S. O. Demokritov, Appl. Phys. Lett. 99, 172501 (2011).

[26] X. Liu, W. Zhang, M. J. Carter, and G. Xiao, J. Appl. Phys. 110, 033910 (2011).

[27] M. P. R. Sabino, S. T. Lim, and M. Tran, Appl. Phys. Express 7, 093002 (2014).

[28] M. Belmeguenai, H. Tuzcuoglu, M. S. Gabor, T. Petrisor, Jr., C. Tiusan, D. Berling, F. Zighem, T. Chauveau, S. M. Chérif, and P. Moch, Phys. Rev. B 87, 184431 (2013).

[29] B. A. Kalinikos, M. P. Kostylev, N. V. Kozhus', and A. N. Slavin, J. Phys.: Condens. Matter 2, 9861 (1990).

[30] M. Haidar, M. Bailleul, M. Kostylev, and Y. Lao, Phys. Rev. B 89, 094426 (2014).

[31] R. F. Soohoo, Phys. Rev. 131, 594 (1963).

[32] R. L. Stamps and B. Hillebrands, Phys. Rev. B 44, 12417 (1991).

[33] R. W. Damon and J. R. Eshbach, J. Phys. Chem. Solids 19, 308 (1961).

[34] Y. Roussigné, F. Ganot, C. Dugautier, P. Moch, and D. Renard, Phys. Rev. B 52, 350 (1995).

[35] H. T. Nembach, J. M. Shaw, M. Weiler, E. Jué, and T. J. Silva, arXiv: 1410.6243

[36] K. Fukumoto, W. Kuch, J. Vogel, J. Camarero, S. Pizzini, F. Offi, Y. Pennec, M. Bonfim, A. Fontaine, and J. Kirschner, J. Magn. Magn. Mater. 293, 863 (2005).

[37] J. M. Shaw, H. T. Nembach, T. J. Silva, and C. T. Boone, J. Appl. Phys. 114, 243906 (2013).

[38] S. Mizukami, Y. Ando, and T. Miyazaki, J. Magn. Mater. 226, 1640 (2001).

[39] F. Fohr, S. Kaltenborn, J. Hamrle, H. Schultheiß, A. A. Serga, H. C. Schneider, B. Hillebrands, Y. Fukuma, L. Wang, and Y. Otani, Phys. Rev. Lett. 106, 226601 (2011).

[40] M. Heide, G. Bihlmayer, and S. Blügel, Phys. Rev. B 78 , 140403(R) (2008). 